\title{
Linx
}

Revue des linguistes de l'université Paris X Nanterre

49 | 2003

L'actualité des notions d'interlangue et d'interaction exolingue

\section{Communication exolingue et Interlangue}

\section{Ulrich Dausendschön-Gay}

\section{(2) OpenEdition}

\section{Journals}

Édition électronique

URL : http://journals.openedition.org/linx/531

DOI : $10.4000 /$ linx.531

ISSN : 2118-9692

Éditeur

Presses universitaires de Paris Nanterre

\section{Édition imprimée}

Date de publication : 1 décembre 2003

Pagination : 41-50

ISSN : 0246-8743

\section{Référence électronique}

Ulrich Dausendschön-Gay, « Communication exolingue et Interlangue », Linx [En ligne], 49 | 2003, mis en ligne le 04 mars 2011, consulté le 10 décembre 2020. URL : http://journals.openedition.org/linx/ 531 ; DOI : https://doi.org/10.4000/linx.531 


\title{
Communication exolingue et Interlangue
}

\author{
Ulrich Dausendschön-Gay \\ Université de Bielefeld
}

\section{Quelques présupposés méthodologiques d'une approche interactionniste}

\subsection{L'exemple de la communication interculturelle}

Dans leur contribution à une publication sur les stéréotypes nationaux dans des discours interethniques, Reinhold Schmitt et Inken Keim (1995) ont critiqué ce qu'ils appellent la «logique déductive» (Subsumtionslogik, en allemand) ${ }^{1}$. Elle présupposerait qu'un entretien entre interlocuteurs d'origines culturelles et linguistiques différentes serait automatiquement une situation de communication interculturelle. Pour l'analyse des données conversationnelles, cette préconception des caractéristiques prétendues intrinsèques de l'échange entraînerait une interprétation qui décrit les données en termes d'interculturalité sans être capable de montrer exactement si un événement perturbateur prend vraiment son origine dans ce domaine spécifique de facteurs qui influent sur la situation et le comportement des interactants. Sur la base de ses origines ethnométhodologiques, l'analyse conversationnelle propose de qualifier un épisode interactionnel selon le principe des «catégories de(s) participants » (member categories, en anglais): ce sont les interactants qui se manifestent mutuellement leur interprétation de la situation. Si, pour eux, la diversité des perspectives culturelles est pertinente pour l'interaction en cours, ils vont le montrer à travers leurs actions verbales et non-verbales. Dans un de nos textes, nous avons pu faire l'analyse d'événements de ce type :

\footnotetext{
1 «Très souvent, dans les recherches sur la communication interculturelle, la diversité des arrièrefonds culturels est considérée comme ayant une pertinence immédiate - voire négative - pour la communication. La preuve empirique de l'interculturalité est cherchée dans les formes spécifiques de malentendus et de problèmes d'intercompréhension ainsi que dans leur fréquence. Ce faisant, les chercheurs introduisent la catégorie d'interculturel comme un présupposé de l'analyse effective de l'interaction. Par conséquent, les problèmes de l'interaction sont interprétés comme étant de nature interculturelle. » (Schmitt/Keim 1995 : 413-414 ; traduit par UDG). C’est dans le cadre général de ce type d'approche conversationnaliste et interactionniste que s'inscrivent les recherches que nous menons à Bielefeld.
} 
- Les explications de faits politiques, géographiques ou autres qui présupposent l'ignorance de la part d'interlocuteurs qui n'ont pas fait l'école française et qui ne vivent pas régulièrement en France; ces épisodes interculturels concernent le problème du savoir commun présupposé (du « common ground ») et ils anticipent des problèmes d'intercompréhension.

- Ces situations risquent parfois de dégénérer en situations de dominance communicative quand l'autochtone exploite l'incompétence présumée de son interlocuteur alloglotte pour établir une asymétrie des places et des rôles.

- Le versant positif de la gestion de ces situations, par contre, exploite au maximum la diversité des perspectives et des points de vue pour enrichir la situation communicative.

Si l'on accepte cette position méthodologique de l'analyse conversationnelle, on sera obligé de ne pas qualifier une interaction comme étant interculturelle mais de constater que les interactants, selon leurs besoins communs ou individuels, définissent des moments/épisodes de l'interaction pendant lesquels la divergence des savoirs culturels peut ou doit être mise en pertinence.

Cela entraine des conséquences pour l'analyse, qui distinguera alors systématiquement entre

- des critères internes, c'est-à-dire les activités de mise en pertinence des interlocuteurs qui se manifestent mutuellement le sens et l'objectif de ce qu'ils sont en train de faire : comme ces critères ne sont que rarement communiqués au moment de l'interaction par les interlocuteurs eux-mêmes, ils ne nous sont pas accessibles directement ;

- des critères externes, c'est-à-dire les notions, théoriquement fondées et appliquées selon une méthodologie de recherche, que les observateurs développent et emploient pour décrire ce qui se passe dans une interaction.

Par conséquent, je propose de qualifier le terme d'interculturel comme une catégorie d'observation ${ }^{2}$ tout en souscrivant à la remarque de Gülich et Mondada (2001 : 201) qui précisent que « la particularité de l’approche ethnométhodologique est que la méthodologie n'est jamais autonome par rapport à l'analyse de cas concrets ». Faire l'analyse de la dimension interculturelle d'une interaction est le résultat d'un choix théorique et méthodologique qui introduit une perspective spécifique par

\footnotetext{
${ }^{2}$ Il est vrai que la fameuse «mentalité ethnométhodologique » propose de développer les notions descriptives et interprétatives des observateurs à partir de l'observation des interactions authentiques. Mais il faut constater que la position extrême selon laquelle le travail de l'observateur consiste à reconstruire fiablement la perspective des interactants en se servant exclusivement de leurs catégories est théoriquement intenable. La deuxième génération des conversationnalistes a développé une position méthodologique qui correspond à celle que je viens de défendre et qui se rapproche de la conception de «l'observateur de deuxième degré » de la théorie des systèmes du sociologue allemand Niklas Luhmann. Voir également Dausendschön-Gay et Krafft 1998.

Gülich et Mondada (2001: 202), dans une des plus claires présentations récentes de l'analyse conversationnelle, tout en défendant les aspects positifs de la position des conversationnalistes de la première génération, décrivent les problèmes méthodologiques en ces termes : « Ce regard [selon le principe de l'unmotivated examination] a souvent été accusé de "naïveté » : cette critique sous-estime le fait qu'adopter cette posture requiert un travail et une rigueur analytique accrus qui amènent à réfléchir sur les catégories que nous avons constamment tendance à projeter sur les données. »
} 
rapport aux données qui sont le point de départ de l'activité des chercheurs. C’est ainsi que se construit l'objet d'une recherche.

\subsection{La notion d'exolingue}

Les principes méthodologiques que je viens d'exposer à l'exemple de l'interculturel s'appliquent également à l'analyse de ce qu'on a l'habitude d'appeler la communication exolingue. Il est vrai que, selon la définition proposée à l'origine par Porquier, la qualité exolingue d'une conversation réside dans le fait de l'inégalité des compétences linguistiques des interlocuteurs par rapport à la langue de la communication en cours. On peut donc supposer que les traces de cette inégalité, aussi minimes qu'elles soient, sont constamment présentes pour les interactants. Cela distingue, à première vue, l'exolingue de l'interculturel. Mais il est difficile de mesurer l'impact des ces traces d'inégalité sur la communication et les interactants peuvent facilement ne pas les tenir en considération. Par conséquent, il est approprié de constater que l'exolingue est une perspective de recherche de l'observateur extérieur et qu'il n'est ni une qualité intrinsèque de l'événement communicationnel ni une donnée empirique à priori. C'est bien dans ce sens que Porquier ${ }^{3}$ a essayé de clarifier quelques questions terminologiques :

On voit $[\ldots]$ que la distinction entre situation exolingue et communication exolingue apparait pertinente. On a d'une part des paramètres qui renvoient aux deux axes (endolingue/exolingue et unilingue/bilingue) comme composantes de la situation; d'autre part des instances de communication (des « événements langagiers », pour de Pietro 1988) qui, de façon variable, mobilisent et actualisent ces paramètres. La compétence bilingue respective des interlocuteurs n'est pas un prédicteur de la nature unilingue/bilingue de l'échange, mais l'un des paramètres de l'observation.

(Porquier 1994 : 166)

Je vais expliquer ma propre perspective, qui radicalise d'une certaine façon le point de vue de Porquier, à l'aide de quelques exemples dont le premier est extrait de la conversation dans un café entre deux collègues de travail.

\footnotetext{
3 Dans son remarquable texte de 1994, il passe en revue le débat terminologique qu'il a déclenché lui-même avec ses textes fondateurs de 1979 et 1984. Il met en rapport les continuums exolingue/endolingue et unilingue/bilingue, il propose de distinguer situation exolingue et communication exolingue et il intègre des réflexions sur la notion d'interlangue, tout en rappelant les cinq paramètres qui, selon lui, permettent de décrire l'exolingue : «Les langues ou idiomes connus des participants, le milieu linguistique de l'interaction, le cadre situationnel de l'interaction, le type d'interaction, le contenu de l'interaction». (Porquier 1994 : 164). Dans ce contexte, et de façon absolument convaincante, Lüdi/Py 2002 (en particulier dans le chapitre 6) intègrent leurs observations sur l'alternance codique (le «code-switching») dans le débat autour des formes et fonctions du choix linguistique dans des situations plurilingues.
} 
Ulrich Dausendschön-Gay

Exemple 1 « tu connais le basque » 4

Interlocuteurs : Jean-Philippe (J, français) et Mikkel (M, danois)

$\mathrm{J}$ : après/après les soirées de rugby là'. $\mathrm{y}$ a toujours une comment ça s'appelle une grosse/ une grosse fête

à paris quoi' . dans le paris'/ . tu sais au sud de paris dans le paris treizième là' . $<M$ :

$a b>$ et eh c'est une soirée basque tu connais le basque non' le pays basque'

$\mathrm{M}$ : oui'

$\mathrm{J}:$ tu vois le pays basque est séparé entre . le sud de la france

$\mathrm{M}$ :

ouais ouais,

$\mathrm{J}:$ et eh le nord de l'espagne

M : $\quad$ ouais ouais c'est espagnol

$\mathrm{J}$ : quoi mais c'est bon, .. et eh c'est des gens qui font pas mal la fête' .. et là' y a une soirée basque a paris dans un gymnase . ou y aura chais pas énormément de/ énormément de personnes quoi' .. eh euh c'est vraiment pas mal quoi,..

$\mathrm{M}: \mathrm{ah}<J: h m>$ et le treize/ treizième c'est . eh : .. bon eh : arrondissement , . pour les riches,

$\mathrm{J}:$ non ça c'est le SEIZième ça,

$\mathrm{M}:$ le seizième,

$\mathrm{J}:$. mais le treizième c'est pas forcément pour les riches ouais, . $\langle M: a b>$ c'est le quartier chinois en fait,

$\mathrm{M}:$ ah ouais, . ouais d'accord, euh . ne/ na/ nation,

$\mathrm{J}:<2$ sec $>$ non place d'Italie

$\mathrm{M}:$ place d'Italie, ouais, sud de nation,

$\mathrm{J}:$ ouais sud de nation ouais,

$\mathrm{M}$ : ouais

$\mathrm{J}$ :. et eh ouais c'est/ c'est pas mal ces soirées là, . tu sors eh pas facile facile mais c'est pas mal ouais,

On peut observer dans l'extrait un certain nombre d'activités qui attirent notre attention de linguistes et qu'on qualifierait d'inhabituelles par rapport à nos expériences d'une communication prétendue normale. On mentionnerait :

- les commentaires métadiscursifs de Jean-Philippe,

- les explications pour paris et le pays basque - inutiles d'ailleurs, comme l'indique Mikkel,

- les petites pauses fréquentes qui précédent les initiatives thématiques de JeanPhilippe qui en assure la presque totalité,

- les difficultés de Mikkel de se prononcer,

- mais aussi l'absence totale de corrections ou d'irritations (à l'exception, peut-être,

\footnotetext{
${ }^{4}$ L'extrait est tiré du corpus de Tim Wahlstätt, enregistré en France en février 2002. Il s'agit de la conversation entre Jean-Philippe L. et son collègue Mikkel B., d'origine danoise et travaillant à Paris depuis septembre 2001.
} 
des pauses) de la part de Jean-Philippe qui fait siennes les paroles de Mikkel pour confirmer la compréhension (sud de nation).

On peut faire l'hypothèse que cette conversation est vécue comme un peu difficile par les participants qui vont attribuer les sources de ces difficultés aux compétences réduites de l'interlocuteur alloglotte. Mais ce qu'on peut observer avant tout dans l'interaction, c'est la construction commune d'une conversation qui réussit à tous les niveaux : progression thématique, intercompréhension, sauvegarde des faces, confirmation du rapport social ${ }^{5}$. Dans la perspective des interactants, la question de savoir si la situation ou la communication est exolingue semble être de moindre intérêt; ce qui importe, c'est leur capacité de gérer, chacun de son côté, les compétences qui permettent de construire ensemble un monde social. Quant aux méthodes (au sens ethnométhodologique du terme) que mettent en œuvre les interactants pour gérer la situation difficile, elles sont les mêmes que celles que nous trouvons dans d'autres types de communication difficile, comme par exemple entre adultes et enfants, entre experts et non-experts ou entre enseignants et élèves. Décrire la spécificité de l'exolingue par rapport à ces autres types de difficulté n'est pas facile au seul niveau linguistique ${ }^{6}$.

Toutes les manifestations de difficultés linguistiques que nous venons de rencontrer dans l'extrait précédent sont absentes dans l'exemple suivant où la seule trace d'alloglossie est le petit accent de la jeune étudiante allemande, Hannelore. Elle vient rendre au directeur d'un foyer d'étudiants la clé de la chambre d'une de ses compatriotes qui est rentrée en Allemagne, mais son principal intérêt est bien sûr de récupérer la caution. Le directeur n'est pas très content, il ne veut pas rendre la caution sans papier signé de la main de la locutrice, et de plus il trouve dans ses notes le nom d'une autre étudiante allemande qui lui doit deux loyers et qu'il prend pour celle qui veut quitter le foyer à l'aide de son amie Hannelore. La confusion est totale, Hannelore et le directeur se perdent dans la jungle des différents noms allemands (Siebrecht, Brünger, etc) que le directeur n'arrive pas à bien articuler et le conflit se développe.

Exemple 2 chez, le directeur du foyer 7

Interlocuteurs : Directeur (D , français), Hannelore $(\mathbf{H}$, allemande)

$\mathrm{D}$ : je ne peux pas rendre les clés’ à une personne que je connais pas ! . vous je vous connais pas,

$\mathrm{H}$ : oui

$\mathrm{D}$ : je peux pas vous rendre l'argent,

$\mathrm{H}$ : oui bien sûr,

\footnotetext{
${ }^{5}$ Pour justifier cette analyse en détail, il suffirait de suivre toute la conversation, qui dure plus d'une heure. A mentionner en particulier le ton très amical de l'échange.

${ }^{6}$ Pour le détail de nos analyses de ces ethnométhodes, $c$. une longue série de publications résumées dans Dausendschön-Gay 1995.

${ }^{7}$ L'extrait est tiré du corpus Bielefeld du projet « Situations de contacts franco-allemands » (Gülich, Krafft, Dausendschön-Gay).
} 
Ulrich Dausendschön-Gay

$\mathrm{D}$ : i faut que ce soit madame eh/ et moi (... ?) moi je connais qu'elle' moi, <bruit> . voilà .. et alors là' il faut $\mathrm{v} / \mathrm{v} /$ vous veniez avec une lettre écrite ! . signée par elle'

$\mathrm{H}: \underline{\text { non mais elle }}<$ soupire $>$

$\mathrm{D}$ : je peux pas' m/ . vous êtes marrant vous, <bruit $>$

$\mathrm{H}$ : vous me laissez expliquer : ‘

D : OUI : 'mais je vous laisse expliquer oui, moi je peux pas, . oui'

$\mathrm{H}$ : alors le problème c'est que: . euh Elle' furchner . elle est partie définitivement en Allemagne, . et l'autre . Brünger' . /brungère/' comme vous dites . elle est partie en Allemagne aussi' mais elle va revenir le d/ TROIS ou le quatre avril,

$\mathrm{D}$ : ah' ben qu'elle vienne le trois ou le quatre' hein . <en articulant $>$ moi' . je peux pas rendre' les clés' à une personne que je connais pas,

$\mathrm{H}$ : ça je comprends bien,

$\mathrm{D}:$ j'ai pas le droit

Au moment où le conflit s'aggrave, Hannelore rappelle au directeur son incompétence linguistique à articuler correctement les noms propres allemands. Ce faisant, elle rétablit l'équilibre rituel des deux protagonistes, chacun a son petit accent. Les esprits se calment après cette thématisation de la dimension interculturelle - et exolingue - qui était sous-jacente à la situation. Loin d'être une donnée de cette communication, la dimension « inter » est une possibilité à exploiter pour des raisons stratégiques. En l'occurrence, il s'agit bien d'une négociation des places et des rôles interactionnels plutôt que d'une trace du caractère exolingue de la communication.

Ces quelques remarques aux sujet des exemples, qui mériteraient plus d'attention, permettent de préciser deux aspects d'analyse qui résultent de notre approche interactionniste :

- Pour les interactants, la problématique principale des communications difficiles est la mise en œuvre de méthodes interactives qui leur permettent de gérer la situation. La divergence des compétences linguistiques, difficile à mesurer et à saisir dans un entretien, est un facteur parmi d'autres qui influe sur le choix des méthodes, mais elle ne les détermine pas. Les verbalisations approximatives de l'interlocuteur danois Mikkel (exemple 1), par exemple, ne sont ni corrigées ni thématisées par son interlocuteur français, et la fréquence des activités d'ajustement linguistique ne semble pas perturber le déroulement thématique de la conversation.

- Parmi les facteurs influant sur le choix des méthodes, il est difficile d'opérationnaliser la distinction nette entre linguistique (exolingue) et interculturel, comme l'a montré le deuxième exemple. Il me semble, par ailleurs, que le besoin de cette distinction est né du côté des linguistes et non des interlocuteurs, natifs ou nonnatifs, experts ou non-experts, adultes ou enfants.

\section{La spécificité des corpus analysés}

Le monde communicationnel que je viens d'évoquer - avec ces partenaires collaborant à la construction d'un monde social et coopérant à la gestion d'une 
situation difficile - est très harmonieux et reflète une spécificité des corpus que nous avons recueillis pour nos recherches sur les «situations de contact entre locuteurs français et allemands $»^{8}$. Pour disposer d'une base empirique suffisamment riche et aussi peu préconstruite que possible, nous avons demandé à des étudiants ou élèves de faire, pendant leurs séjours linguistiques en France (études, cours de langue, visite chez des correspondants), des enregistrements de leurs entretiens quotidiens, à savoir des conversations à table avec des amis ou dans leurs familles, des demandes de renseignements dans la rue, à la banque ou aux services de la municipalité ${ }^{9}$. Dans chacun des ces enregistrements, nous avons affaire à une situation qui met en contact des personnes qui se respectent mutuellement, qui acceptent les contrats de communication (rendre service, donner un renseignement, bavarder) et qui poursuivent communément les buts de leur interaction. Les interlocuteurs arrivent avec des degrés variés d'uni-, de bi- ou de multilinguisme, ils exploitent au maximum les ressources de la situation pour gérer la communication et les obstacles qui se produisent.

Les alloglottes de nos corpus, majoritairement des étudiants ou des élèves, se trouvent plutôt en situation de tourisme linguistique ou de séjour d'études que dans une situation de migration ${ }^{10}$. Leurs langues et cultures d'origine jouissent d'un statut haut dans la société d'accueil, leur altérité est soit perçue comme minime, soit connotée positivement. Toute communication étant située, les événements que nous analysons dans nos études doivent être compris comme des manifestations et constructions locales de ces estimations et évaluations réciproques. En particulier les situations dans les familles sont très particulières puisque le motif du séjour est bien l'amélioration des compétences linguistiques des jeunes étrangers. Il en résulte

- un choix obligatoire du français ou de l'allemand comme langue de communication, les mélanges (des "parlers bilingues ») et l'alternance codique n'étant pas choisis ;

- une répartition de rôles interactionnels de tuteur et d'apprenant qui permettent un ménagement particulier des faces ;

- un éventail d'activités spécifiques, comme des hétéro-réparations fréquentes, des commentaires linguistiques, des séquences d'apprentissage, des séquences latérales explicites et expansives, etc.

\footnotetext{
${ }^{8}$ Au début de nos recherches, dans les années 80 du siècle dernier, nous avons employé cette notion qui évite provisoirement les connotations et implications méthodologiques du terme d'exolingue. Il faut, cependant, noter que nous avons adopté plus tard dans nos publications le terme exolingue que nous continuons d'employer comme un raccourci qui facilite la communication avec nos collègues.

${ }^{9}$ Chacun des enregistrements (à l'heure actuelle plus de 80 , dans des situations très diverses et avec un grand éventail de types de participants) est accompagné d'un questionnaire sur les données de la situation et les interactants.

${ }^{10}$ L'ampleur des différences est manifeste quand on compare la situation de nos étudiants avec celle des participants au projet européen sur l'acquisition des langues par des migrants (Perdue 1992) ou avec les descriptions que donnent Georges Lüdi et Bernard Py dans la deuxième édition (2002) de leur étude modèle de l' « être bilingue » (par exemple p. 18).
} 


\section{La dimension interactionnelle de l'interlangue}

Nos observations sur les méthodes dont se servent les interactants pour gérer une situation plurilingue ont déclenché des recherches sur la modélisation du rapport entre interaction sociale et acquisition qui s'inscrivent dans un courant interactionniste général ${ }^{11}$. Les concepts de SPA (Séquences Potentiellement Acquisitionnelles) et de contrat didactique (proposé par de Pietro, Matthey et Py) ou notre idée du SLASS (Second Language Acquisition Support System) ont été largement acceptés comme point de départ des réflexions nécessaires qui doivent compléter les études sur les lectes d'apprenants $^{12}$. Cependant, les considérations relatives aux contextes d'apprentissage, au caractère situé de toute cognition (et de toute acquisition) et à la spécificité des situations plurilingues des corpus étudiés appellent à une grande prudence pour toute généralisation de résultats d'analyse dans le monde social très particulier, harmonieux et marqué de coopérativité permanente de la part de tous les interactants dans les familles de nos étudiants et élèves. Les remarques de Mondada et Pekarek-Doehler $(2000: 166 / 7)$ à ce propos sont très stimulantes quand elles résument la conception des cognitions situées - elles sont situées dans l'organisation locale de la pratique sociale, dans un contexte socio-historique, et dans la distribution des cognitions entre les acteurs humains et les objets - pour en tirer la conclusion suivante : "Ces définitions du caractère situé de la cognition permettent de montrer qu'elle se manifeste dans des compétences qui sont imbriquées dans des activités et des cadres de participations spécifiques et qui donc ne peuvent pas être 'exportées' sans problème, généralisées à d'autres contextes ou traitées comme universelles. (ibid. 169)»

Il en résulte que la notion d'interlangue, habituellement liée au développement systématique (et probablement universel pour certains domaines) des compétences d'un individu apprenant une langue, doit être reconsidérée dans la perspective du caractère situé de toute action. La diversité des contextes de pratique sociale demande et stabilise des compétences d'action qui permettent à l'individu apprenant de répondre aux exigences habituelles des interactions quotidiennes. Ces compétences sont sociales, il est par conséquent difficile de les décrire en faisant abstraction du contexte de leur déploiement; il semble inapproprié de les concevoir comme étant prioritairement des compétences de l'individu qui par ailleurs dispose d'une grande variabilité de compétences selon les situations et les interactants; la diversité et les différences des compétences entre individus dans leurs capacités d'action s'expliquent par la spécificité des contextes d'appropriation et des pratiques sociales dans lesquelles elles émergent, se développent et se stabilisent selon les besoins.

\footnotetext{
11 Pour un résumé $c f$. Pekarek-Doehler 2000 et Dausendschön-Gay (sous presse).

12 Cf. Perdue 2000 : 301 : "The results of the original [ESF] project (summarized in Perdue 1993) clearly identify a limited set of organizing principles of different levels - syntactic, semantic, and pragmatic (in the sense of the principles organizing coherent discourse) - that structure learner varieties. These principles interact, and the specific interaction determines the organization of a given learner variety at a given time. Development can be understood as a reorganization of the variety following a change in the relative weight of the contributing principles."
} 
Pour exemplifier les conséquences d'une telle approche, je reviens à un des aspects de la particularité de nos corpus, c'est-à-dire des pratiques sociales qu'ils documentent. Les étudiants et élèves faisant un séjour linguistique en France ou en Allemagne ont conclu avec la majorité de leurs interlocuteurs habituels une sorte de contrat didactique qui, par moments, semble l'emporter sur tout autre contrat de communication. La fréquence des centrations sur la forme ${ }^{13}$ constitue en même temps une préférence d'activités qui sont censées contribuer à la construction de ce que Py appelle la microsyntaxe ${ }^{14}$; elle concerne la structure interne des segments du discours, elle est analytique et s'ouvre directement sur la notion de correction grammaticale. Pour Py, son développement est ultérieur à l'émergence de la macrosyntaxe qui relie des unités, porteuses de sens discursif, selon les règles d'une «logique d'implication pratique » et qui accumule au fur et à mesure du développement d'une interaction la mémoire discursive des acteurs. La macrosyntaxe repose massivement sur l'emploi de structures figées, inanalysées et ayant le statut de simples lexèmes même si leur structure interne peut être décrite comme étant composée et syntaxiquement complexe; elle permet à l'apprenant, en particulier dans les premier stades acquisitionnels, de participer à la praxis sociale. On voit à l'exemple de Mikkel que ce système est très performant sans la moindre intervention au niveau de la microsysntaxe.

\section{Perspectives}

Par conséquent, il n'est pas sûr que la centration sur la forme et son rôle facilitateur pour des processus du développement de l'interlangue explique, de façon générale, le fonctionnement de l'appropriation d'une langue seconde en interaction sociale. Il faut plutôt faire l'hypothèse que la présence d'un contrat didactique fait partie d'une pratique sociale spécifique qui favorise le développement de la microsyntaxe et qui s'inscrit dans le cadre plus large de l'enseignement des langues à l'école ${ }^{15}$. Même si, à long terme, Mikkel ('alloglotte du premier exemple) va être capable de se débrouiller aussi bien que Hannelore (exemple 2) dans son conflit avec le directeur du foyer, rien ne prouve que les processus qui l'y ont conduit aient été les mêmes. C'est autour de ce type de questionnements que la recherche interactionniste sur l'acquisition va continuer.

u.daugay@uni-bielefeld.de

\footnotetext{
${ }^{13}$ Je me sers ici du terme introduit par Pierre Bange dans le contexte de ses réflexions sur la bifocalisation.

${ }^{14}$ Py reprend une idée de Berrendonner et de Béguelin, qui nous rappelle aussi les conceptions de la grammaire du français parlé de Blanche-Benveniste et de son équipe aixoise: "Au niveau microsyntaxique les unités lexicales sont organisées selon une logique de rection: des marques grammaticales expriment les relations qui existent entre les unités lexicales (par exemple la détermination ou la coréférence). Chaque réseau de relations constitue une clause, sorte d'ilot grammaticalisé, pouvant d'ailleurs, dans certains cas, se réduire à sa plus simple expression, à savoir un seul morphème. Les clauses sont reliées les unes aux autres au niveau macrosyntaxique selon une logique d'implication pratique. Chaque clause apporte une information à la mémoire discursive des partenaires engagés dans l'interaction. » (Py $2002: 3$ ).

15 Cf. À ce propos les remarques très éclairantes de Gabriele Pallotti 2002.
} 
Ulrich Dausendschön-Gay

\section{BIBLIOGRAPHIE}

DAUSENDSCHÖN-GAY, U. (1995) : «La gestion interactionnelle de la différence des compétences linguistiques : le cas des interactions exolingues », Cabiers de praxématique $25,31-51$.

DAUSENDSCHÖN-GAY, U. (sous presse) : «Producing and learning to produce utterances in social interaction ", in EUROSLA Yearbook 3, 2003.

DAUSENDSCHÖN-GAY, U. \& KRAFFT, U. (1998) : «Quand l'exolingue devient de l'interculturel », LIDIL 18, 93-111.

GÜLICH, E. \& MONDADA, L. (2001) : « Analyse conversationnelle», in HOLTUS, G. et al. (éds.) : Lexikon der Romanistischen Linguistik. Volume I-2. Tübingen, Niemeyer, 196-250.

KRAFFT, U. \& DAUSENDSCHÖN-GAY, U. (1994) : «Analyse conversationnelle et recherche sur l'acquisition », dans PY, B. (dir.) : L'acquisition d'une langue seconde. Quelques développements théoriques récents. Bulletin VALS/ASLA 59, 127-158.

LÜDI, G. \& PY, B. (2002) : Etre bilingue [Deuxième édition revue]. Bern, Peter Lang.

MONDADA, L. \& PEKAREK-DOEHLER, S. (2000) : «Interaction sociale et cognition située : quels modèles pour la recherche sur l'acquisition des langues ?", Acquisition et Interaction en Langue Étrangère 12, 147-174.

PALLOTTI, G. (2002) : «La classe dans une perspective écologique de l'acquisition», Acquisition et Interaction en Langue Etrangère 16, 165-197.

PEKAREK-DOEHLER, S. (2000) : «Approches interactionnistes de l'acquisition des langues étrangères : concepts, recherches, perspectives", Acquisition et Interaction en Langue Étrangère 12, 3-26.

PERDUE, C., ed. (2000) : "Introduction to "The structure of learner varieties" ", Studies in Second Language Acquisition 22, 299-305.

PORQUIER, R. (1994) : "Communication exolingue et contextes d'appropriation: le continuum acquisition/apprentissage », dans PY, B. (dir.): L'acquisition d'une langue seconde. Quelques développements théoriques récents. Bulletin VALS/ASLA 59, 159-170.

PY, B. (2002) : « Acquisition d'une langue seconde, organisation macrosyntaxique et émergence d'une microsyntaxe », Marges linguistiques 3.

SCHMITT, R. \& KEIM, I. (1995) : « Das Problem der subsumtionslogischen Konstitution von Interkulturalität », in CZYZEWSKI, M. et al. (eds). Nationale Selbst- und Fremdbilder im Gespräch. Opladen, Westdeutscher Verlag, 413-429. 